\title{
Institucionalização e atividade física em idosos e suas relações com medo de cair e qualidade de vida
}

\author{
Institutionalization and physical activity in the elderly and their relationships \\ with fear of falling and quality of life
}

\author{
Tamara Ferreira Rachadel', Juliana Broering², Marcelo Luza ${ }^{3}$, Lisiane Piazza $₫$ \\ ${ }^{1}$ Fisioterapeuta, graduada pelo Instituto de Ensino Superior da Grande Florianópolis (IESGF). Florianópolis, SC. \\ 2 Acadêmica do curso de Fisioterapia do IESGF. Florianópolis, SC. \\ ${ }_{3}^{3}$ Fisioterapeuta, graduado pela Universidade de Passo Fundo (UPF). Especialista em Terapia Manual e Postural pelo Centro Universitário de Maringá (CESUMAR). \\ Aperfeiçoamento em Osteopatia em andamento na Escuela de Osteopatia de Madrid. Campinas, SP. \\ ${ }^{4}$ Docente do curso de Fisioterapia do IESGF e da Universidade do Estado de Santa Catarina (UDESC). Florianópolis, SC.
}

\section{RESUMO}

Objetivos: Avaliar a associação da institucionalização e da prática de atividade física com o medo de cair e a qualidade de vida de idosos.

Métodos: Foram selecionados para este estudo 61 idosos, divididos em três grupos: 21 institucionalizados, 20 não institucionalizados ativos e 20 não institucionalizados não ativos. Foram utilizados como instrumentos o questionário FES-I para avaliar o medo de cair e o questionário SF-36 para avaliação da qualidade de vida. Os participantes foram recrutados em instituições de longa permanência e entre idosos da comunidade, residentes na Grande Florianópolis. Os dados foram analisados por meio de estatística descritiva e inferencial (Anova One Way, testes de Kruskall-Wallis e U de Mann-Whitney) com nível de significância de $\mathrm{p} \leq 0,05$.

Resultados: No questionário FES-I houve diferença significativa entre os idosos institucionalizados e não institucionalizados ativos, com maior pontuação nos institucionalizados $(30 \pm 10,5 ; \mathrm{p}=0,02)$, significando maior preocupação com quedas. Na avaliação da qualidade de vida foi observada diferença significativa entre os grupos no domínio capacidade funcional, com maior pontuação nos não institucionalizados ativos $(78 \pm 19,6)$, seguidos pelos não institucionalizados não ativos $(51 \pm 27,3)$ e institucionalizados $(35,9 \pm 25,9)(p=0,001)$. No domínio dor, a maior pontuação (menos dor) foi nos institucionalizados $(83,2 \pm 21,6)$, seguidos pelos não institucionalizados ativos $(61,4 \pm 25,9)$ e não institucionalizados não ativos $(54,4 \pm 33,3)(\mathrm{p}=0,005)$.

Conclusões: Nos idosos incluídos neste estudo, a institucionalização associou-se a maior preocupação com quedas, menor capacidade funcional e menos queixas de dor. A prática de atividade física associou-se ao melhor desempenho no domínio capacidade funcional do questionário sobre qualidade de vida e à menor preocupação com quedas.

DESCRITORES: idoso; saúde do idoso; institucionalização; qualidade de vida.

\section{ABSTRACT}

Aims: To assess the association of institutionalization and of the practice of physical activity with the fear of falling and the quality of life in elderly.

Methods: Sixty-one elderly individuals were selected and classified into three groups: 21 institutionalized, 20 non-institutionalized active, and 20 non-institutionalized not active. The FES-I and the SF-36 questionnaires were used to evaluate fear of falling and quality of life, respectively. Participants were recruited in long term care facilities and among the elderly of the community, all residents of the metropolitan area of Florianópolis, Brazil. Data were analyzed using descriptive and inferential statistics (Anova One Way, Kruskal-Wallis test and Mann-Whitney) with a significance level $\mathrm{p} \leq 0.05$.

Results: No significant difference was found in the FES-I questionnaire among the institutionalized and non-institutionalized active elderly, with the highest score in the institutionalized $(30 \pm 10.5 ; \mathrm{p}=0.02)$, meaning greater concern about falls. Quality of life was significant different between the groups in the area functional capacity, with higher scores in non-institutionalized active (78 \pm 19.6$)$, followed by non-institutionalized not active $(51 \pm 27.3)$ and institutionalized ones $(35.9 \pm 25.9)(\mathrm{p}=0.001)$. In the pain domain, the highest score (less pain) was found in the institutionalized (83.2 \pm 21.6$)$, followed by non-institutionalized active $(61.4 \pm 25.9)$ and non-institutionalized not active ones $(54.4 \pm 33.3)$ $(\mathrm{p}=0.005)$.

Conclusions: In the elderly included in this study, institutionalization was associated with greater concern about falls, less functional capacity, and fewer complaints of pain. Physical activity was associated with better performance in the functional capacity domain of the quality of life questionnaire, and with less concern about falls.

KEY WORDS: aged; health of the elderly; institutionalization; quality of life. 
Abreviaturas: INST, grupo de idosos institucionalizados; NINST-A, grupo de idosos não institucionalizados ativos; NINST-NA, grupo de idosos não institucionalizados não ativos; SF-36, questionário de qualidade de vida 36-Item Short Form Health Survey; FES-I, escala para avaliação de risco de queda Falls Efficacy Scale-International; PSN, escore de qualidade de vida pelo instrumento Perfil de Saúde de Nottingham.

\section{INTRODUÇÃO}

O envelhecimento conduz a diversas alterações físicas, como o aumento na rigidez articular, bem como nos tendões e ligamentos, redução na velocidade de condução nervosa, além de menor flexibilidade, mobilidade articular, perda de equilíbrio, coordenação e agilidade. Adicionalmente, pode levar a alterações na massa óssea e muscular, aumentando dessa forma o risco de quedas, sendo a prática de atividades físicas uma forma de minimizar essas perdas $[1,2]$.

A qualidade de vida na população idosa está diretamente relacionada à sua capacidade de manter a autonomia e a independência. Para isso, é essencial a manutenção de um estilo de vida saudável. Sendo assim, a orientação e o acompanhamento por profissionais da saúde quanto à prática de atividades físicas são de extrema importância, uma vez que estas estimulam a manutenção e a recuperação das funções vitais do organismo, além de auxiliar nas funções cerebrais e musculoesqueléticas $[3,4]$.

A população idosa vem crescendo gradativamente no Brasil. Em 1991, os idosos representavam 4,8\% da população do país e em 2000 representavam 5,9\%, passando para $7,4 \%$ em 2010, sendo esse aumento mais expressivo nas regiões sul e sudeste $[5,6]$. Esse crescimento da população idosa tem ocasionado um aumento das instituições de longa permanência, as quais surgem como uma alternativa de suporte social, já que a falta de tempo para cuidar do idoso leva muitas famílias a optarem por sua institucionalização. Porém, transferir um idoso de sua casa para uma instituição de longa permanência pode levá-lo à depressão, sensação de isolamento e separação da sociedade, além de conduzir a um menor nível de atividade física, podendo interferir também na sua qualidade de vida [7-11].

Diante do exposto, este estudo teve como objetivo analisar a influência da institucionalização e da prática de atividade física sobre o medo de cair e a qualidade de vida de idosos.

\section{MÉTODOS}

O presente estudo caracterizou-se como transversal descritivo e comparativo, sendo aprovado pelo Comitê de Ética e Pesquisa da Universidade Paulista sob

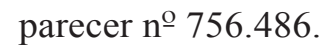

Foram incluídos no estudo 61 idosos com idade igual ou acima de 60 anos, de ambos os sexos, divididos em três grupos: 21 idosos institucionalizados (grupo INST), 20 idosos não institucionalizados ativos (grupo NINST-A) e 20 não institucionalizados não ativos (grupo NINST-NA).

Todos os participantes foram escolhidos de forma não probabilística intencional. O cálculo amostral, com base em estudo piloto prévio, foi realizado através do programa GPower (v.3.1), considerando um poder de teste de $95 \%$ e tamanho do efeito (mínima mudança a ser detectada entre os grupos) de 0,5 .

Os critérios de inclusão para todos os grupos foram idade acima de 60 anos, ausência de depressão e função cognitiva preservada, com capacidade para compreender e responder às questões referentes aos instrumentos utilizados na pesquisa. Além destes, para o grupo INST, residir em uma instituição de longa permanência das cidades da Grande Florianópolis; para o grupo NINST-A, não residir em instituição de longa permanência e praticar 150 minutos ou mais por semana de atividade física de intensidade moderada ou vigorosa; e para o grupo NINST-NA, não residir em instituição de longa permanência e praticar menos de 150 minutos por semana de atividade física de intensidade moderada ou vigorosa.

A classificação dos idosos em ativos e não ativos baseou-se nas recomendações do American College of Sports Medicine e da American Heart Association, que classificam como ativos os indivíduos que praticam até 150 minutos por semana de atividade física de intensidade no mínimo moderada [12]. A classificação do grau de atividade física foi feita pela análise dos dados coletados na ficha de avaliação dos sujeitos.

A coleta de dados dos idosos institucionalizados foi feita em instituições de longa permanência da Grande Florianópolis, após contato prévio com os responsáveis pelas instituições, solicitando autorização para realização do estudo e agendamento das avaliações. Os idosos dos outros dois grupos foram selecionados por conveniência, entre os moradores com mais de 60 anos que residiam nos municípios da Grande Florianópolis. Todos os idosos foram informados sobre os objetivos e procedimentos da pesquisa e assinaram um termo de consentimento livre e esclarecido concordando com a participação no estudo.

O período de coleta de dados foi entre agosto e setembro de 2014. Os instrumentos utilizados foram uma ficha para caracterização dos participantes, elaborada pelos pesquisadores, o questionário de 
qualidade de vida 36-Item Short Form Health Survey (SF-36) e a escala para avaliação de risco de queda Falls Efficacy Scale-International (FES-I). O SF-36, um questionário usado para avaliação da qualidade de vida, é traduzido e validado para a língua portuguesa [13]. É composto por 11 questões e 36 itens que englobam oito componentes (domínios ou dimensões), representados por capacidade funcional (dez itens), aspectos físicos (quatro itens), dor (dois itens), estado geral da saúde (cinco itens), vitalidade (quatro itens), aspectos sociais (dois itens), aspectos emocionais (três itens), saúde mental (cinco itens) e uma questão comparativa sobre a percepção atual da saúde e há um ano. $\mathrm{O}$ indivíduo recebe um escore em cada domínio, que varia de 0 a 100 , sendo 0 o pior escore e 100 o melhor.

A FES-I, utilizada para medir o medo de cair em idosos, também é traduzida e validada para a população brasileira. Essa escala questiona, através de 16 domínios, o quanto o indivíduo está preocupado em sofrer quedas ao realizar determinadas atividades. Cada domínio pode receber uma pontuação de 1 (não estou preocupado) a 4 (muito preocupado) em relação ao risco de quedas. A preocupação relaciona-se com o histórico de quedas, de forma que uma pontuação $\geq 23$ e $<30$ pontos sugere uma associação com histórico de quedas esporádicas, já uma pontuação $\geq 31$ pontos sugere uma associação com quedas recorrentes [14].

Os dados foram analisados pelo Statistical Package for the Social Sciences (versão 15.0), sendo utilizada a estatística descritiva para caracterização dos sujeitos, o teste de Shapiro Wilk para verificar a normalidade dos dados, o Anova One Way com post hoc de Tukey para verificar a existência de diferenças entre os sujeitos quanto à idade, massa e estatura, o teste de Kruskall Wallis para comparar o medo de cair e os domínios do SF-36 (capacidade funcional, aspectos físicos, dor, estado geral, vitalidade, aspectos sociais, aspectos emocionais, saúde mental) entre o INST, NINST-A e NINST-NA. Em caso de diferença significativa no Kruskall Wallis foi utilizado o teste U Mann-Whitney como post hoc com correção de Bonferroni. O nível de significância adotado foi $\mathrm{p} \leq 0,05$.

\section{RESULTADOS}

Na Tabela 1 são apresentadas as características dos participantes quanto à idade, massa, estatura, tempo de prática de atividade física por semana, tempo de institucionalização e histórico de quedas. Em relação à idade, a maior média foi a do grupo institucionalizado e a menor foi a do grupo não institucionalizado ativo $(p=0,001)$. Em relação à atividade física, o grupo INST teve a classificação semelhante à do NINSTNA, ou seja, foi classificado como não ativo de acordo com o estabelecido na metodologia. O grupo institucionalizado apresentou maior histórico de quedas ( $76 \%$ dos participantes), enquanto o grupo não institucionalizado ativo informou o menor histórico de quedas (65\% dos participantes). A Tabela 2 apresenta as principais doenças relatadas pelos participantes dos três grupos.

Tabela 1. Características dos idosos institucionalizados (INST), não institucionalizados ativos (NINST-A) e não institucionalizados não ativos (NINST-NA), quanto a idade, peso, estatura, prática de atividade física, tempo de institucionalização e histórico de quedas. Grande Florianópolis, SC, agosto-setembro de 2014.

\begin{tabular}{|c|c|c|c|c|c|}
\hline & $\begin{array}{c}\text { INST } \\
(n=21)\end{array}$ & $\begin{array}{l}\text { NINST-A } \\
(n=20)\end{array}$ & $\begin{array}{c}\text { NINST-NA } \\
(n=20)\end{array}$ & $F^{\#}$ & $\mathrm{p}$ \\
\hline Idade (anos) & $78,5 \pm 8,02^{+}$ & $67,9 \pm 5,6^{t \neq}$ & $75 \pm 9,5^{\ddagger}$ & 9,57 & $0,001^{*}$ \\
\hline Peso (kg) & $69,2 \pm 11,8$ & $70,6 \pm 14,2$ & $67,5 \pm 13,1$ & 0,27 & 0,76 \\
\hline Estatura $(\mathrm{cm})$ & $159,9 \pm 10,2$ & $160,6 \pm 7,2$ & $159,1 \pm 11,4$ & 0,11 & 0,88 \\
\hline Atividade física (min/sem) & $78,1 \pm 36,9$ & $621,1 \pm 161,3$ & $76,5 \pm 52,3$ & & \\
\hline Tempo de institucionalização (meses) & $39,1 \pm 32,9$ & & & & \\
\hline Histórico de quedas & $\begin{array}{l}\text { Sim: } 16(76 \%) \\
\text { Não: } 5(24 \%)\end{array}$ & $\begin{array}{l}\text { Sim: } 13(65 \%) \\
\text { Não: } 7(35 \%)\end{array}$ & $\begin{array}{l}\text { Sim: } 15(75 \%) \\
\text { Não: } 5(25 \%)\end{array}$ & & \\
\hline
\end{tabular}

INST: idosos institucionalizados; NINST-A: idosos não institucionalizados ativos; NINST-NA: idosos não institucionalizados e não ativos; $\mathrm{min} / \mathrm{sem}$ : minutos por semana.

\# Valor da estatística (Anova One Way).

* Diferença significativa pela Anova One way.

+ Diferença significativa pelo Post Hoc de Tukey entre o INST e NINST-A.

‡ Diferença significativa pelo Post Hoc de Tukey entre o NINST-A e o NINST-NA. 
Tabela 2. Doenças referidas por idosos institucionalizados, não institucionalizados ativos e não institucionalizados não ativos. Grande Florianópolis, SC, agosto-setembro de 2014.

\begin{tabular}{|c|c|c|c|}
\hline Doença & $\begin{array}{c}\text { INST } \\
(n=21)\end{array}$ & $\begin{array}{l}\text { NINST-A } \\
(n=20)\end{array}$ & $\begin{array}{l}\text { NINST-NA } \\
(\mathbf{n}=20)\end{array}$ \\
\hline Hipertensão arterial sistêmica & $9(43 \%)$ & $14(70 \%)$ & $16(80 \%)$ \\
\hline Diabetes & $4(19 \%)$ & $5(25 \%)$ & $8(40 \%)$ \\
\hline Deficiência visual & $18(86 \%)$ & $13(65 \%)$ & $16(80 \%)$ \\
\hline Deficiência auditiva & $8(38 \%)$ & $2(10 \%)$ & $6(30 \%)$ \\
\hline Cardiopatia & $4(19 \%)$ & $3(15 \%)$ & $12(60 \%)$ \\
\hline Alteração na coluna & $8(38 \%)$ & $12(60 \%)$ & $9(45 \%)$ \\
\hline Alteração vascular/linfática & $8(38 \%)$ & 7 (35\%) & $9(45 \%)$ \\
\hline
\end{tabular}

INST: idosos institucionalizados; NINST-A: idosos não institucionalizados ativos;

NINST-NA: idosos não institucionalizados e não ativos.

Tabela 3. Resultados da Escala de Eficácia de Quedas (FES-I), demonstrando a preocupação em sofrer quedas nos três grupos de idosos (mais de 60 anos): institucionalizados, não institucionalizados ativos e não institucionalizados não ativos. Grande Florianópolis, SC, agosto-setembro de 2014.

\begin{tabular}{c|c|c|c|c|c} 
& & $\begin{array}{c}\text { INST } \\
(\mathbf{n = 2 1 )}\end{array}$ & $\begin{array}{c}\text { NINST-A } \\
(\mathbf{n = 2 0})\end{array}$ & $\begin{array}{c}\text { NINST-NA } \\
(\mathbf{n = 2 0 )}\end{array}$ & $\mathbf{p}$ \\
\hline \multirow{2}{*}{ FES-I } & $\mathbf{M} \pm \mathbf{D P}$ & $30 \pm 10,5^{\#}$ & $21,3 \pm 8,6^{\#}$ & $27,2 \pm 14,7$ & $0,02^{*}$ \\
\hline
\end{tabular}

INST: idosos institucionalizados; NINST-A: idosos não institucionalizados ativos; NINST-NA: idosos não institucionalizados e não ativos; FES-I: Escala de Eficácia de Quedas M: média; IC: intervalo de confiança.

* Diferença significativa pelo teste de Kruskall Wallis.

\# Diferença significativa pelo teste U Mann Whitney entre o INST e NINST-A.

Na Tabela 3 são apresentados os resultados relativos à preocupação com quedas nos três grupos. Os três apresentaram pontuação menor que 31 , sendo o INST o grupo com maior pontuação $(30 \pm 10,5)$ e o NINST-A o grupo com menor pontuação $(21,3 \pm 8,6)$. A diferença foi significativa entre INST e NINST-A, com maior medo de cair nos idosos institucionalizados em relação aos não institucionalizados ativos. Já os idosos não institucionalizados não ativos tiveram pontuação intermediária, não diferindo significativamente dos outros grupos (Tabela 3).

$\mathrm{Na}$ Tabela 4 são apresentados os resultados relativos ao questionário de qualidade de vida SF-36, sendo observada diferença significativa na capacidade funcional entre os grupos INST e NINST-A e entre os grupos NINST-A e NINST-NA, com maior pontuação no NINST-A nos dois casos, ou seja, os idosos ativos tiveram maior capacidade funcional, tanto em relação aos institucionalizados quanto aos não institucionalizados inativos.

No domínio dor, a maior pontuação (menos queixa de dor) foi nos idosos institucionalizados $(83,2 \pm 21,6)$, seguidos pelos não institucionalizados ativos $(61,4 \pm 25,9)$ e não institucionalizados não ativos $(54,4 \pm 33,3)$ $(p=0,005)$. Os outros domínios não apresentaram diferenças significativas entre os grupos (Tabela 4).

\section{DISCUSSÃO}

Através da aplicação da FES-I observou-se maior medo de cair nos idosos institucionalizados quando comparados com os não institucionalizados que praticam atividade física. Sendo assim, os resultados do presente estudo sugerem que a combinação de institucionalização com inatividade poderia levar a um maior medo de cair e consequente risco de quedas, uma vez que, quando a institucionalização e a prática de atividade física foram analisadas isoladamente nas comparações entre o INST e NINST-NA (ambos grupos inativos, sendo a institucionalização apenas que os diferenciava) e entre o NINST-A e NINST-NA (ambos não institucionalizados, sendo a prática de atividade física o que os diferenciava), não se observou diferença entre os grupos.

O medo de cair pode ser identificado como um fator de risco para redução na qualidade de vida, restrição das atividades, redução da independência e risco de quedas na população idosa [15]. Segundo Amador et al. [16], o medo de cair pode estar associado ao avanço da idade, déficits de equilíbrio e diminuição da força muscular, levando à restrição da atividade física e interferindo na independência do idoso. Adicionalmente, Gonçalves et al. [17], afirmam que os idosos que vivem em asilos, 
Tabela 4. Resultados do questionário de qualidade de vida SF-36 aplicado aos três grupos de idosos (mais de 60 anos): institucionalizados, não institucionalizados ativos e não institucionalizados não ativos. Grande Florianópolis, SC, agosto-setembro de 2014.

\begin{tabular}{|c|c|c|c|c|c|}
\hline & & $\begin{array}{c}\text { INST } \\
(\mathbf{n}=21)\end{array}$ & $\begin{array}{l}\text { NINST-A } \\
(\mathbf{n}=\mathbf{2 0})\end{array}$ & $\begin{array}{l}\text { NINST-NA } \\
(\mathbf{n}=\mathbf{2 0 )}\end{array}$ & $\mathbf{p}$ \\
\hline Capacidade funcional & $\begin{array}{l}M \pm D P \\
\text { IC } 95 \%\end{array}$ & $\begin{array}{c}35,9 \pm 25,9^{+} \\
24,4-49,1\end{array}$ & $\begin{array}{c}78 \pm 19,6^{+\neq} \\
68,8-87,2\end{array}$ & $\begin{array}{l}51 \pm 27,3^{*} \\
38,2-63,8\end{array}$ & $0,001^{*}$ \\
\hline Aspectos físicos & $\begin{array}{l}M \pm D P \\
\text { IC } 95 \%\end{array}$ & $\begin{array}{c}79,7 \pm 33,1 \\
62,3-94,5\end{array}$ & $\begin{array}{c}62,5 \pm 39,3 \\
44,1-80,1\end{array}$ & $\begin{array}{c}52,5 \pm 41,2 \\
33,1-71,8\end{array}$ & 0,07 \\
\hline Dor & $\begin{array}{l}M \pm D P \\
I C 95 \%\end{array}$ & $\begin{array}{c}83,2 \pm 21,6^{+} \S \\
74,3-94,5\end{array}$ & $\begin{array}{c}61,4 \pm 25,9^{+} \\
49,3-73,5\end{array}$ & $\begin{array}{c}54,4 \pm 33,3^{5} \\
38,8-70\end{array}$ & $0,005^{*}$ \\
\hline Estado geral & $\begin{array}{l}M \pm D P \\
I C 95 \%\end{array}$ & $\begin{array}{c}68,1 \pm 20,5 \\
57,6-76,9\end{array}$ & $\begin{array}{c}68,4 \pm 22,2 \\
58,1-78,9\end{array}$ & $\begin{array}{c}58,7 \pm 29,9 \\
44,7-72,7\end{array}$ & 0,52 \\
\hline Vitalidade & $\begin{array}{l}\mathrm{M} \pm \mathrm{DP} \\
\mathrm{IC} 95 \%\end{array}$ & $\begin{array}{l}70,7 \pm 12,4 \\
64,8-76,7\end{array}$ & $\begin{array}{c}69,7 \pm 19,7 \\
60,5-78,9\end{array}$ & $\begin{array}{c}66 \pm 23,4 \\
55-77\end{array}$ & 0,75 \\
\hline Aspectos sociais & $\begin{array}{l}\mathrm{M} \pm \mathrm{DP} \\
\mathrm{IC} 95 \%\end{array}$ & $\begin{array}{c}95,8 \pm 4,4 \\
90,2-100\end{array}$ & $\begin{array}{c}85 \pm 23,5 \\
74-96\end{array}$ & $\begin{array}{c}78,7 \pm 30,6 \\
64,4-93,1\end{array}$ & 0,13 \\
\hline Aspectos emocionais & $\begin{array}{l}\mathrm{M} \pm \mathrm{DP} \\
\text { IC } 95 \%\end{array}$ & $\begin{array}{c}87,3 \pm 12,8 \\
72,9-100\end{array}$ & $\begin{array}{c}73,3 \pm 35,2 \\
56,8-89,8\end{array}$ & $\begin{array}{c}76,6 \pm 34,3 \\
60,6-92,7\end{array}$ & 0,26 \\
\hline Saúde mental & $\begin{array}{l}M \pm D P \\
\text { IC } 95 \%\end{array}$ & $\begin{array}{c}78,1 \pm 24,2 \\
66,4-89,6\end{array}$ & $\begin{array}{c}77,2 \pm 19,6 \\
67,9-86,4\end{array}$ & $\begin{array}{r}69 \pm 27,9 \\
55,9-82,1\end{array}$ & 0,48 \\
\hline
\end{tabular}

INST: idosos institucionalizados; NINST-A: idosos não institucionalizados ativos; NINST-NA: idosos não institucionalizados e não ativos.

* Diferença significativa pelo teste de Kruskall Wallis.

+ Diferença significativa pelo teste U Mann Whitney entre o INST e NINST-A.

₹ Diferença significativa pelo teste U Mann Whitney entre NINST-A e NINST-NA.

$\S$ Diferença significativa pelo teste U Mann Whitney entre INST e NINST-NA.

casas geriátricas e clínicas são mais sedentários e menos autônomos, o que contribui para o aumento nas morbidades relacionadas à autonomia, sendo as quedas um dos principais agravos.

Silva et al. [18] observaram menor desempenho funcional em idosas com preocupação com quedas. Segundo os autores, a preocupação em cair pode conduzir a alterações na biomecânica da marcha, com alterações na velocidade, deslocamento no centro de massa e outros fatores, o que pode aumentar o risco de quedas.

Pela classificação da pontuação da FES-I, escores maiores ou iguais a 23 pontos sugerem associação com quedas esporádicas e maior ou igual a 31 pontos sugerem uma associação com quedas recorrentes $[14,16]$, significando que existe relação entre receio de cair e histórico de quedas. No presente estudo confirmamse essas relações, pois o INST foi o grupo com maior receio e maior histórico de quedas, enquanto o NINST-A foi o grupo com menor receio e menor histórico de quedas.

A prática de atividade física diária contribui para a prevenção de quedas, pois pode aumentar significativamente a força e manter a composição e o peso corporal eficientes para a locomoção, além de contribuir para a melhora do equilíbrio [19]. Estas afirmações corroboram os resultados do estudo de
Padoin et al. [20], os quais observaram que idosos sedentários apresentam menor mobilidade funcional, maiores déficits no equilíbrio e mais alterações na marcha, quando comparados a idosos que praticavam exercício físico regularmente.

No presente estudo, a maioria dos idosos institucionalizados relataram já ter sofrido alguma queda e apresentaram maior medo de cair em relação aos não institucionalizados, corroborando com Gonçalves et al. [17], os quais afirmam que a prevalência de quedas entre institucionalizados é alta. Acredita-se também que o maior medo de cair nos idosos institucionalizados deste estudo possa ter relação com o fato desse grupo ser mais velho em comparação aos não institucionalizados ativos, uma vez que o medo de cair e o risco de quedas aumentam com o avançar da idade [21].

Segundo Minayo et al. [22], a reincidência de quedas entre os idosos é comum, o que pode gerar incapacidades parciais ou dependência, afetando negativamente sua qualidade de vida. Além disso, a qualidade de vida do idoso pode diminuir ainda mais quando este possui problemas de saúde, os quais se tornam mais frequentes com o avançar da idade. A qualidade de vida caracteriza-se como um aspecto multidimensional que não deve ser tratado de forma generalizada. É um conceito amplo que tem por 
objetivo analisar, mais do que a condição clínica de saúde do indivíduo, os aspectos ligados à percepção do bem-estar pessoal e autoestima [23].

No presente estudo, a qualidade de vida foi analisada através do questionário SF-36, sendo constatado que dentre os oito domínios analisados, houve diferença entre os grupos somente nos domínios capacidade funcional e dor. $\mathrm{Na}$ capacidade funcional houve diferença significativa entre os grupos INST e NINST-A, NINST-A e NINST-NA, com maior pontuação no NINST-A, demonstrando que os idosos ativos conseguem realizar suas atividades de vida diária de maneira mais independente. No domínio dor, observou-se diferença significativa entre os grupos INST e NINST-A, e INST e NINST-NA, com maior pontuação no INST, o que significa que os idosos do grupo INST tinham uma menor intensidade de dor que influencia nas atividades da vida diária. Acreditase que essa menor intensidade na dor dos idosos institucionalizados possa ser explicada pelo fato da dor ser uma sensação subjetiva [24] e que talvez os idosos do grupo INST tivessem fácil acesso a medicamentos analgésicos quando sentissem dor, uma vez que eram amparados constantemente por profissionais da saúde.

A prática regular de atividade física está associada a melhora na saúde, aumento da funcionalidade e maior longevidade, sendo amplamente incentivada entre os idosos, uma vez que pode reverter ou minimizar as alterações decorrentes do processo de envelhecimento, contribuindo para manutenção da funcionalidade e da qualidade de vida dessa população [25-30]. Mota et al. [31], ao comparar a qualidade de vida entre participantes de programas de atividade física e não participantes, observou no grupo que praticava exercícios um desempenho superior em todos os domínios do questionário SF-36 comparado com o grupo que não praticava, demonstrando que a atividade física é um importante fator para o aumento da qualidade de vida.

Perlini et al. [32] afirmam que o fato de morar só, para o idoso, tem sido associado a menor qualidade de vida, agravo da morbidade e, até mesmo, indicador de risco de mortalidade. Nas palavras dessas autoras, diante das dificuldades que surgem no cotidiano familiar de garantir o cuidado e a qualidade de vida do idoso, a família e, muitas vezes, o próprio idoso, visualizam a institucionalização como uma alternativa viável.

O estudo de Mincato et al. [33], realizado somente com idosos institucionalizados, identificou uma correlação inversa significativa entre o escore de capacidade funcional pelo instrumento Atividades Básicas da Vida Diária e o escore de qualidade de vida pelo instrumento Perfil de Saúde de Nottingham (PSN), ou seja, quanto melhor a capacidade funcional, melhor foi a qualidade de vida (menor foi o escore do teste PSN). No presente estudo identificou-se uma capacidade funcional menor nos idosos institucionalizados quando comparados aos não institucionalizados ativos. Podese inferir, portanto, que sendo a capacidade funcional um importante componente da qualidade de vida, os participantes do grupo INST apresentavam de certa forma uma interferência na sua qualidade de vida. Provavelmente as instituições visitadas para o presente estudo forneçam um bom amparo aos idosos, os quais, entretanto, por terem uma média de idade maior e/ou por realizarem menos atividade física, apresentam diminuição em sua capacidade funcional.

Foram consideradas limitações do presente estudo o fato de não haver idosos considerados ativos nas instituições de longa permanência para poder comparar aos outros grupos, e a dificuldade de aplicação do SF-36 na população idosa, uma vez que o questionário é extenso e possui perguntas semelhantes. Considerase também uma limitação a média de idade maior dos idosos institucionalizados, o que pode ter causado um viés nos resultados. Sugerem-se futuros estudos que avaliem idosos institucionalizados que sejam ativos e não ativos, para que se possa verificar a influência da prática de atividade física na qualidade de vida e medo de cair na população institucionalizada.

Sendo assim, os resultados do presente estudo, nas condições avaliadas, mostram que a institucionalização associou-se a maior preocupação com quedas, menor capacidade funcional e menos queixas de dor, enquanto a prática de atividade física associou-se à melhor pontuação no domínio capacidade funcional da qualidade de vida, assim como à menor preocupação com quedas.

\section{REFERÊNCIAS}

1. Matsudo SM, Matsudo VKR, Neto TLB. Impacto do envelhecimento nas variáveis antropométricas, neuromotoras e metabólicas da aptidão física. Rev Bras Ciênc Mov. 2000;8(4):21-32.

2. Shepard RJ. Physical activity, fitness and health: the current consensus. Quest. 1995;47(3):228-303.

3. Freitas MC, Queiroz TA, Sousa JAV. O significado da velhice e da experiência de envelhecer para os idosos. Rev Esc Enf USP. 2010;44(2):407-12. http://dx.doi.org/10.1590/S0080-62342010000200024

4. Netto FLM. Aspectos biológicos e fisiológicos do envelhecimento humano e suas implicações na saúde do idoso. Pensar a Prática. 2004;7:75-84. 
5. Instituto Brasileiro de Geografia e Estatística (IBGE). Primeiros resultados definitivos do Censo 2010. [Internet]. [cited 2014 November 18]. Available from: http://censo2010.ibge.gov.br/noticias-censo?busca=1\&idnoticia $=1866 \& \mathrm{t}=$ primeiros-resultados-definitivos-censo2010-populacao-brasil-190-755-799-pessoas\&view=noticia

6. Pereira RJ, Cotta RMM, Franceschini SCC, Ribeiro RCL, Sampaio RF, Priore SE, Cecon PR. Contribuição dos domínios físico, social, psicológico e ambiental para a qualidade de vida global de idosos. Rev Psiquiatr RS. 2006;28(1):27-38. http://dx.doi.org/10.1590/s010181082006000100005

7. Pestana LC, Santo FHE. As engrenagens da saúde na terceira idade: um estudo com idosos asilados. Rev Esc Enf USP. 2008;42(2):268-75. http://dx.doi.org/10.1590/S0080-62342008000200009

8. Tier CG, Fontana RT, Soares NV. Refletindo sobre idosos institucionalizados. Rev Bras Enferm. 2004;57(3):332-5. http://dx.doi. org/10.1590/S0034-71672004000300015

9. Araujo MOPH, Ceolim MF. Avaliação do grau de independência de idosos residentes em instituições de longa permanência. Rev Esc Enf USP. 2007;41(3):378-85. http://dx.doi.org/10.1590/S0080-62342007000300006

10. Washburn RA, McAuley E, Katula J, Mihalko SL, Boileau RA. The Physical Activity Scale for Elderly (PASE): evidence for validity. J Clin Epidemiol. 1999;52(7):643-51. http://dx.doi.org/10.1016/S0895-4356(99)00049-9

11. Buchman AS, Boyle, PA, Wilson RS, Bienias JL, Bennett DA. Physical activity and motor decline in older persons. Muscle Nerve. 2007;35:354-62. http://dx.doi.org/10.1002/mus.20702

12. Nelson ME, Rejeski WJ, Blair SN, Duncan PW, Judge JO, King AC. Physical Activity and Public Health in Older Adults - Recommendation from the American College of Sports Medicine and the American Heart Association. Circulation. 2007;116:1094-105. http://dx.doi org/10.1161/CIRCULATIONAHA.107.185650

13. Ciconelli RM, Ferraz MB, Santos W, Meinão I, Quaresma MR. Tradução para língua portuguesa e validação e do questionário genérico de avaliação de qualidade de vida SF-36. Rev Bras Reumatol. 1999;39(3):143-50.

14. Camargos FFO, Dias RC, Dias JMD, Freire MTF. Adaptação transcultural e avaliação das propriedades psicométricas da Falls Efficacy Scale - International em idosos brasileiros (FES-I - BRASIL). Rev Bras Fisioter. 2010;14(3):237-43. http://dx.doi.org/10.1590/S141335552010000300010

15. Young WR, Williams AM. How fear of falling can increase fall-risk in older adults: Applying psychological theory to practical observations. Gait Posture. 2015;41:7-12. http://dx.doi.org/10.1016/j.gaitpost.2014.09.006

16. Amador LF, Loera JA. Preventing postoperative falls in the older adult. J Am Coll Surg. 2007;204(3):447-53. http://dx.doi.org/10.1016/j jamcollsurg.2006.12.010

17. Gonçalves LG, Vieira ST, Siqueira FV, Hallal PC. Prevalência de quedas em idosos asilados do município de Rio Grande/RS. Rev Saúde Publ. 2008;42(5):938-45. http://dx.doi.org/10.1590/S0034-89102008000500021

18. Silva DO, Pazzinatto MF, Oliveira MC, Aragão FA, Albuquerque CE. Influência da preocupação com quedas na mobilidade e na força de reação do solo em idosas durante descida de escada. Sci Med. 2014;24(4):361-7. http://dx.doi.org/10.15448/1980-6108.2014.4.18539

19. Spirduso WW. Dimensões físicas do envelhecimento. $2^{\mathrm{a}}$ ed. São Paulo: Manole; 2005.

20. Padoin PG, Gonçalves MP, Comaru T, Silva AM. Análise comparativa entre idosos praticantes de exercício físico e sedentários quanto ao risco de quedas. Mundo Saúde. 2010;34(2):158-64.

21. Abreu SSE, Caldas CP. Velocidade de marcha, equilíbrio e idade: um estudo correlacional entre idosas praticantes e idosas não praticantes de um programa de exercícios terapêuticos. Rev Bras Fisioter. 2008;12(4):324-30. http://dx.doi.org/10.1590/S1413-35552008000400012

22. Minayo MCS, Hartz ZA, Buss PM. Qualidade de vida e saúde: um debate necessário. Ciênc Saúde Coletiva. 2000;5(1):7-18. http://dx.doi org/10.1590/S1413-81232000000100002

23. Martins ABT, Soares AP, Barboza MCC, Sales RM, Frota MA, Santos ZMSA. Qualidade de vida em idosos institucionalizados: revisão sistemática. Convibra Saúde - Congresso Virtual Brasileiro de Educação, gestão e promoção da saúde [Internet]. [cited 2014 November 18]. Available from: http://www.convibra.com.br/upload/paper/2012/72/2012_72_4260.pdf

24. 24 Sousa FAEF. Dor: o quinto sinal vital. Rev Latino Am Enferm. 2002; 10(3):446-7. http://dx.doi.org/10.1590/S0104-11692002000300020

25. Stessman JMD, Hammerman RMD, Cohen AMD, Mor EMA, Jacobs JMBBS. Physical Activity, Function and longevity among the very old. Arch Intern Med. 2009;169(16):1476-83. http://dx.doi.org/10.1001/archinternmed.2009.248

26. American College of Sports Medicine, Chodzko-Zajko WJ, Proctor DN, Fiatarone Singh MA, Minson CT, Nigg CR, Salem GJ, Skinner JS. American College of Sports Medicine position stand. Exercise and physical activity for older adults. Med Sci Sports Exerc. 2009;41(7): 1510-30. http://dx.doi.org/10.1249/MSS.0b013e3181a0c95c

27. Juraki D, Pédisic Z, Greblo Z. Physical activity in different domains and health-related quality of life: a population-based study. Qual Life Res. 2010;19(9):1303-9. http://dx.doi.org/10.1007/s11136-010-9705-6

28. Karinkanta S, Nupponen R, Heinonen A, Pasanen M, Sievänen H, Uusi-Rasi K, Fogelholm M, Kannus P. Effects of exercise on healthrelated quality of life and fear of falling in home-dwelling older women. J Aging Phys Act. 2012;20(2):198-214.

29. Borges MRD, Moreira AK. Influências da prática de atividades físicas na terceira idade: estudo comparativo dos níveis de autonomia para o desempenho nas AVDs e AIVDs entre idosos ativos fisicamente e idosos sedentários. Motriz. 2009;15(3):562-73.

30. Okuma SS. O idoso e a atividade física: Fundamentos e pesquisa. Campinas: Papirus; 1998.

31. Mota J, Ribeiro JL, Carvalho J, Matos MG. Atividade física e qualidade de vida associada à saúde em idosos participantes e não participantes em programas regulares de atividade física. Rev Bras Educ Fis Esporte. 2006;20(3):219-25.

32. Perlini NMOG, Leite MT, Furini AC. Em busca de uma instituição para a pessoa idosa morar: motivos apontados por familiares. Rev Esc Enf USP. 2007;41(2):229-36. http://dx.doi.org/10.1590/S0080-62342007000200008

33. Mincato PC, Freitas CLR. Qualidade de vida dos idosos residentes em instituições asilares da cidade de Caxias do Sul-RS. Rev Bras de Ciên do Envelh Hum. 2007;4(1):127-38. 